\title{
MEAN VALUE TYPE INEQUALITIES FOR QUASINEARLY SUBHARMONIC FUNCTIONS
}

\author{
OLEKSIY DOVGOSHEY \\ Institute of Applied Mathematics and Mechanics of NASU, R. Luxemburg Str. 74, Donetsk 83114, Ukraine \\ e-mail: aleksdov@mail.ru \\ and JUHANI RIIHENTAUS \\ Department of Physics and Mathematics, University of Eastern Finland, P.O. Box 111, FI-80101 Joensuu, \\ Finland \\ e-mail: juhani.riihentaus@gmail.com
}

(Received 7 February 2011; accepted 20 September 2012; first published online 25 February 2013)

\begin{abstract}
The mean value inequality is characteristic for upper semi-continuous functions to be subharmonic. Quasinearly subharmonic functions generalise subharmonic functions. We find the necessary and sufficient conditions under which subsets of balls are big enough for the characterisation of non-negative, quasinearly subharmonic functions by mean value inequalities. Similar result is obtained also for generalised mean value inequalities where, instead of balls, we consider arbitrary bounded sets, which have nonvoid interiors and instead of the volume of ball some functions depending on the radius of this ball.
\end{abstract}

1991 Mathematics Subject Classification. Primary 31B05, 31C05; Secondary 31C45.

\section{Subharmonic functions and generalisations. Some definitions and results.}

1.1. Notation. Our notation is rather standard, see for example $[\mathbf{1 1}, \mathbf{1 6}-\mathbf{1 8}]$ and the references therein. If $E \subset \mathbb{R}^{n}$ and $x \in \mathbb{R}^{n}$, then we write $\delta_{E}(x):=\inf \left\{|x-y|: y \in E^{c}\right\}$, where $E^{c}=\mathbb{R}^{n} \backslash E$. The Lebesgue measure in $\mathbb{R}^{n}$ is denoted by $m_{n}$. We write $B^{n}(x, r)$ for the open ball in $\mathbb{R}^{n}$ with centre $x$ and radius $r$. Recall that $m_{n}\left(B^{n}(x, r)\right)=v_{n} r^{n}$, where $v_{n}:=$ $m_{n}\left(B^{n}(0,1)\right)$. We denote by Int $D, \bar{D}$ and $\partial D$ the interior, the closure and the boundary of

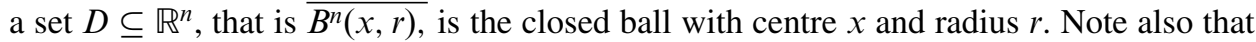
our constants $C$ and $K$ are non-negative, mostly $\geq 1$, and may vary from line to line.

1.2. Subharmonic functions and generalisations. Let $\Omega$ be an open set in $\mathbb{R}^{n}, n \geq$ 2. Let $u: \Omega \rightarrow[-\infty,+\infty)$ be Lebesgue measurable. We adopt the following definitions:

(i) $u$ is subharmonic if $u$ is upper semi-continuous and if

$$
u(x) \leq \frac{1}{v_{n} r^{n}} \int_{B^{n}(x, r)} u(y) d m_{n}(y)
$$

for all balls $\overline{B^{n}(x, r)} \subset \Omega$. A subharmonic function may be $\equiv-\infty$ on any component of $\Omega$ (see [8, p. 9] and [1, p. 60]). 
(ii) $u$ is nearly subharmonic if $u^{+} \in \mathcal{L}_{\text {loc }}^{1}(\Omega)$ and inequality (1) holds for all balls $\overline{B^{n}(x, r)} \subset \Omega$. Observe that this definition is slightly more general than the usual one, compare [17, p. 51] with the standard definition [8, p. 14].

(iii) Let $K \geq 1$. Then, $u$ is $K$-quasinearly subharmonic if $u^{+} \in \mathcal{L}_{\text {loc }}^{1}(\Omega)$ and inequality

$$
u_{M}(x) \leq \frac{K}{v_{n} r^{n}} \int_{B^{n}(x, r)} u_{M}(y) d m_{n}(y)
$$

holds for all $M \geq 0$ and for all balls $\overline{B^{n}(x, r)} \subset \Omega$. Here, $u_{M}:=\max \{u,-M\}+M$. The function $u$ is quasinearly subharmonic if $u$ is $K$-quasinearly subharmonic for some $K \geq 1$. For the definition and properties of quasinearly subharmonic functions, see for example $[\mathbf{5}, \mathbf{9 - 1 1}, \mathbf{1 2}-\mathbf{1 8}]$ and the references therein. We write $Q N S(\Omega)$ for the set of all non-negative quasinearly subharmonic functions on the open set $\Omega \subseteq \mathbb{R}^{n}$.

Proposition 1.3. (cf. [17], Proposition 2.1, pp. 54-55). The following statements hold:

(i) A subharmonic function is nearly subharmonic but not conversely.

(ii) A function is nearly subharmonic if and only if it is 1-quasinearly subharmonic.

(iii) A nearly subharmonic function is quasinearly subharmonic but not conversely.

(iv) If $u: \Omega \rightarrow[0,+\infty)$ is Lebesgue measurable, then $u$ is $K$-quasinearly subharmonic if and only if $u \in \mathcal{L}_{\text {loc }}^{1}(\Omega)$ and

$$
u(x) \leq \frac{K}{m_{n}\left(B^{n}(x, r)\right)} \int_{B^{n}(x, r)} u(y) d m_{n}(y)
$$

for all closed balls $\overline{B^{n}(x, r)} \subset \Omega$.

Note that if $u$ is $K$-quasinearly subharmonic and non-negative in $\Omega$, then (2) holds also for every open ball $B^{n}(x, r) \subseteq \Omega$.

Let $A$ be a subset of the open half-line $(0, \infty)$ such that 0 is a limit point of $A$ and let $u: \Omega \rightarrow[-\infty,+\infty)$ be an upper semi-continuous function on an open set $\Omega \subseteq \mathbb{R}^{n}$. The classical Blascke-Privalov theorem, see for example [2, Chapter II, Section 2], implies that $u$ is subharmonic if inequality (1) holds whenever $r \in A$ and $\overline{B^{n}(x, r)} \subset \Omega$. Moreover, the simple examples show that if non-negative $u \in \mathcal{L}_{\text {loc }}^{1}(\Omega)$, then the fulfilment of (2) for all $(x, r) \in \Omega \times A$ with $\overline{B^{n}(x, r)} \subset \Omega$ does not, generally, imply $u \in Q N S(\Omega)$. A legitimate question to raise in this point is in finding the sets $A \subseteq(0, \infty)$ for which every nonnegative $u \in \mathcal{L}_{\text {loc }}^{1}(\Omega)$ is quasinearly subharmonic if (2) holds for $(x, r) \in \Omega \times A$ whenever $\overline{B^{n}(x, r)} \subset \Omega$.

DEFinition 1.4. Let $\Omega$ be an open set in $\mathbb{R}^{n}$. A set $A \subseteq(0, \infty)$ is favourable for $\Omega$ (favourable for the characterisation of non-negative, quasinearly subharmonic functions in $\Omega$ ) if for every non-negative $u \in \mathcal{L}_{\text {loc }}^{1}(\Omega)$ the following conditions are equivalent:

(i) $u \in Q N S(\Omega)$.

(ii) There is $K=K(u, A, \Omega) \geq 1$ such that for all $x \in \Omega$ the inequality

$$
u(x) \leq \frac{K}{v_{n} r^{n}} \int_{B^{n}(x, r)} u(y) d m_{n}(y)
$$

holds whenever $r \in A$ and $\overline{B^{n}(x, r)} \subset \Omega$. 
We can characterise the favourable subsets of $(0, \infty)$ by the following way.

THEOREM 1.5. The following three statements are equivalent for every $A \subseteq(0, \infty)$ :

(i) $A$ is favourable for all open sets $\Omega \subseteq \mathbb{R}^{n}$.

(ii) The characteristic function

$$
\chi_{\Gamma}(x)= \begin{cases}1 & \text { if } x \in \Gamma \\ 0 & \text { if } x \in \Omega \backslash \Gamma\end{cases}
$$

is quasinearly subharmonic for all open sets $\Omega \subseteq \mathbb{R}^{n}$ and all Lebesgue measurable sets $\Gamma \subseteq \Omega$ if and only if there is a constant $K=K(\Gamma, \Omega, n)$ such that the inequality

$$
m_{n}\left(B^{n}(x, r)\right) \leq K m_{n}\left(\Gamma \cap B^{n}(x, r)\right)
$$

holds for $(x, r) \in \Omega \times A$ whenever $\overline{B^{n}(x, r)} \subset \Omega$.

(iii) There exists $C=C(A)>1$ such that

$$
\left[\frac{x}{C}, x\right] \cap A \neq \varnothing
$$

for every $x \in(0, \infty)$.

We shall prove the equivalence (i) $\equiv$ (iii) in Theorem 2.5 below. Observe also that the implication (i) $\Rightarrow$ (ii) is trivial and that (ii) $\Rightarrow$ (iii) follows directly from the proof of Theorem 2.5. The quasidisks give the important example of the sets $\Gamma$ such that (4) holds in a bounded domain $\Omega \subseteq \mathbb{R}^{2}$ whenever $\overline{B^{2}(x, r)} \subset \Omega$. It is a particular case of the GehringMartio result that proves (4) for the so-called quasiextremal distance domains in $\mathbb{R}^{n}, n \geq 2$ (see [7, Lemma 2.13]).

The following result closely connected with Theorem 1.5, follows from Theorem 2.14 formulated in the second section of the paper.

THEOREM 1.6. Let $f$ be a positive function on $(0, \infty)$. The following three statements are equivalent.

(i) For all open sets $\Omega \subseteq \mathbb{R}^{n}$, Lebesgue measurable functions $u: \Omega \rightarrow[0, \infty)$ are quasinearly subharmonic if and only if there are constants $K=K(u, \Omega, n) \geq 1$ such that

$$
u(x) \leq \frac{K}{(f(r))^{n}} \int_{B^{n}(x, r)} u(y) d m_{n}(y)
$$

for all closed balls $\overline{B^{n}(x, r)} \subset \Omega$.

(ii) For all open sets $\Omega \subseteq \mathbb{R}^{n}$ and all Lebesgue measurable sets $\Gamma \subseteq \Omega$, the characteristic functions $\chi_{\Gamma}$ are quasinearly subharmonic if and only if there are constants $K=$ $K(\Gamma, \Omega, n)$ such that the inequality

$$
(f(r))^{n} \leq K m_{n}\left(B^{n}(x, r) \cap \Gamma\right)
$$

holds for all closed balls $\overline{B_{n}(x, r)} \subset \Omega$ with $x \in \Gamma$.

(iii) There are a set $A \subseteq(0, \infty)$ and a constant $c>1$ such that:

(iii) The inequality $f(r) \leq \mathrm{cr}$ holds for all $r \in(0, \infty)$;

(iii $\left.{ }_{2}\right) \ln A$ is an $\varepsilon$-net in $\mathbb{R}$ for some $\varepsilon>0$; 
(iii) ${ }_{3}$ The inequality

$$
\frac{1}{c} r \leq f(r)
$$

holds for all $r \in A$.

Note that condition (iii) of Theorem 1.5 holds if and only if the $\operatorname{set} \ln (A):=\{\ln x$ : $x \in A\}$ is an $\varepsilon$-net in $\mathbb{R}$ for some $\varepsilon>0$. A characterisation in terms of porosity for the sets $A$, which are favourable for bounded open sets $\Omega \subseteq \mathbb{R}^{n}$, is proved in Theorem 2.12 below.

2. Generalised mean value inequalities. Inequality (2), characteristic for quasinearly subharmonic functions, can be generalised by some distinct ways. Our first theorem characterises non-negative quasinearly subharmonic functions via mean values over some sets more general than just balls.

2.1. Similarities of the Euclidean space. Let $\Omega$ and $D$ be subsets of $\mathbb{R}^{n}$ with marked points $p_{\Omega} \in \Omega$ and $p_{D} \in D$. In what follows, we always suppose that Int $D \neq \emptyset$ and $p_{D} \in$ Int $D$. Denote by $\operatorname{Sim}\left(p_{D}, p_{\Omega}\right)$, the set of all similarities $h: \mathbb{R}^{n} \rightarrow \mathbb{R}^{n}$ such that $h\left(p_{D}\right)=p_{\Omega}$ and $h(D) \subseteq \Omega$. Recall that $h$ is a similarity if there is a positive number $k=k(h)$, the similarity constant of $h$, such that

$$
|h(x)-h(y)|=k|x-y|
$$

for all $x, y \in \mathbb{R}^{n}$. The group of all similarities of the Euclidean space $\mathbb{R}^{n}$ is sometimes denoted as $S M\left(\mathbb{R}^{n}\right)$, see for example [4, 5.1.14], and we also adopt this designation. Observe that each similarity $h \in S M\left(\mathbb{R}^{n}\right)$ can be written in the form

$$
h(x)=k(h) T x+a, \quad x \in \mathbb{R}^{n},
$$

where $k(h)>0$, and $T: \mathbb{R}^{n} \rightarrow \mathbb{R}^{n}$ is an orthogonal linear mapping and $a \in \mathbb{R}^{n}$.

THEOREM 2.2. Let $\Omega$ be an open set in $\mathbb{R}^{n}, n \geq 2$, let $D$ be a bounded, Lebesgue measurable set with the marked point $p_{D} \in$ Int $D$ and let $u: \Omega \rightarrow[0, \infty)$ be a function from $\mathcal{L}_{\text {loc }}^{1}(\Omega)$. Then, $u$ is quasinearly subharmonic if and only if there is $C \geq 1$ such that

$$
u\left(x_{\Omega}\right) \leq \frac{C}{m_{n}(h(D))} \int_{h(D)} u(y) d m_{n}(y)
$$

for every point $x_{\Omega}$ and all $h \in \operatorname{Sim}\left(p_{D}, x_{\Omega}\right)$. If $u$ is $K$-quasinearly subharmonic, then $C=$ $C\left(D, p_{D}, K, n\right)$ and, conversely, if (5) holds, then $u$ is $K$-quasinearly subharmonic with $K=K\left(D, p_{D}, C, n\right)$.

Proof. Write

$$
R_{D}:=\sup _{y \in D}\left|p_{D}-y\right|, \quad \text { and } \quad r_{D}:=\delta_{\operatorname{Int}(D)}\left(p_{D}\right)
$$

Suppose that $u$ is quasinearly subharmonic, that is there is $K \geq 1$ such that (2) holds for all $B^{n}(x, r) \subseteq \Omega$. Let $x_{\Omega}$ be an arbitrary point of $\Omega$ and let $h \in \operatorname{Sim}\left(p_{D}, x_{\Omega}\right)$. The last membership relation implies the inclusions

$$
B^{n}\left(x_{\Omega}, k(h) r_{D}\right) \subseteq \Omega \quad \text { and } \quad h(D) \subseteq \overline{B^{n}\left(x_{\Omega}, k(h) R_{D}\right)}
$$


where $k(h)$ is the similarity constant of $h$. Consequently, we obtain

$$
\begin{aligned}
& \frac{1}{m_{n}(h(D))} \int_{h(D)} u(y) d m_{n}(y) \geq \frac{1}{v_{n}\left(k(h) R_{D}\right)^{n}} \int_{h(D)} u(y) d m_{n}(y) \\
& \geq\left(\frac{r_{D}}{R_{D}}\right)^{n} \frac{1}{v_{n}\left(k(h) r_{D}\right)^{n}} \int_{B^{n}\left(x_{\Omega}, k(h) r_{D}\right)} u(y) d m_{n}(y) \geq\left(\frac{r_{D}}{R_{D}}\right)^{n} \frac{u\left(x_{\Omega}\right)}{K} .
\end{aligned}
$$

Thus, if $f$ is $K$-quasinearly subharmonic, then (5) holds with

$$
C=\frac{K\left(R_{D}\right)^{n}}{\left(r_{D}\right)^{n}}
$$

Conversely, suppose that (5) holds with some $C \geq 1$ for all $x_{\Omega}$ and all $h \in$ $\operatorname{Sim}\left(p_{D}, x_{\Omega}\right)$. Let $B^{n}\left(x_{\Omega}, r_{0}\right) \subseteq \Omega$. Let $h$ be an arbitrary similarity with $k(h)=\frac{r_{0}}{R_{D}}$ and with $h\left(p_{D}\right)=x_{\Omega}$. Then, we have $h \in \operatorname{Sim}\left(p_{D}, x_{\Omega}\right)$ and $B^{n}\left(x_{\Omega}, k(h) r_{D}\right) \subseteq h(D) \subseteq \overline{B^{n}\left(x_{\Omega}, r_{0}\right)}$. Consequently,

$$
\begin{aligned}
& \frac{C}{m_{n}\left(B^{n}\left(x_{\Omega}, r_{0}\right)\right)} \int_{B^{n}\left(x_{\Omega}, r_{0}\right)} u(y) d m_{n}(y) \geq \frac{C m_{n}(h(D))}{m_{n}\left(B^{n}\left(x_{\Omega}, r_{0}\right)\right) m_{n}(h(D))} \int_{h(D)} u(y) d m_{n}(y) \\
& \geq u\left(x_{\Omega}\right) \frac{m_{n}(h(D))}{m_{n}\left(B^{n}\left(x_{\Omega}, r_{0}\right)\right)}
\end{aligned}
$$

Since

$$
\frac{m_{n}\left(B^{n}\left(x_{\Omega}, r_{0}\right)\right)}{m_{n}(h(D))}=\frac{v_{n}\left(r_{0}\right)^{n}}{m_{n}(h(D))}=\frac{v_{n}(k(h))^{n}\left(R_{D}\right)^{n}}{(k(h))^{n} m_{n}(D)}=\frac{v_{n} R_{D}^{n}}{m_{n}(D)},
$$

inequality (1) holds with

$$
K=C \frac{v_{n} R_{D}^{n}}{m_{n}(D)}
$$

REMARK 2.2.1. The standard notion of quasinearly subharmonicity is defined by the condition (5), where $D=B^{n}(0,1), p_{D}=0$ and the considered similarities $h$ are of the form $h(x)=r_{0} x+x_{\Omega}$. The point of Theorem 2.2 is that the definition and its consequences are, however, much more general: instead of just $D=B^{n}(0,1)$ and $p_{D}=0$, one may consider arbitrary bounded sets $D$ with non-void interior Int $D$ and an arbitrary $p_{D} \in \operatorname{Int} D$

REMARK 2.2.2. Inequality (5) remains valid for each non-negative quasinearly subharmonic function if we use bi-Lipschitz mappings $h$ instead of similarities, but in this more general case the constant in (5) depends on the Lipschitz constant of $h$ (see Lemma 2.1 in [6]).

Inequality (5) remains also valid for unbounded sets $D$ if $m_{n}(D)<\infty$.

Proposition 2.3. Let $\Omega$ be an open set in $\mathbb{R}^{n}, n \geq 2$, $D$ a Lebesgue measurable set with $m_{n}(D)<\infty, p_{D}$ a point of $\operatorname{Int}(D)$ and let $u: \Omega \rightarrow[0, \infty)$ be a $K$-quasinearly subharmonic function. Then, there is a constant $C=C\left(D, p_{D}, K, n\right)$ such that (5) holds for all $x_{\Omega} \in \Omega$ and $h \in \operatorname{Sim}\left(p_{D}, x_{\Omega}\right)$. 
Proof. If $D$ is bounded, then this proposition follows from Theorem 2.2. Suppose $D$ is an unbounded. Let $t>1$ be a constant. It is easy to show that there is a ball $B^{n}\left(p_{D}, r_{t}\right)$ with a sufficiently large radius $r_{t}$ such that

$$
\operatorname{tm}\left(D \cap B^{n}\left(p_{D}, r_{t}\right)\right) \geq m_{n}(D) .
$$

Write

$$
D_{t}:=D \cap B^{n}\left(p_{D}, r_{t}\right) \quad \text { and } \quad p_{D_{t}}:=p_{D} .
$$

Note that $D_{t}$ satisfies all conditions of Theorem 2.2 and that $p_{D_{t}} \in \operatorname{Int}\left(D_{t}\right)$. Consequently, there is $K \geq 1$ such that the inequality

$$
u\left(x_{\Omega}\right) \leq \frac{K}{m_{n}\left(h\left(D_{t}\right)\right)} \int_{h\left(D_{t}\right)} u(y) d m_{n}(y)
$$

holds for all $x_{\Omega}$ and $h \in \operatorname{Sim}\left(p_{D_{t}}, x_{\Omega}\right)$. If $h \in \operatorname{Sim}\left(p_{D}, x_{\Omega}\right)$, then we have $h \in \operatorname{Sim}\left(p_{D_{t}}, x_{\Omega}\right)$ and $h\left(D_{t}\right) \subseteq h(D)$. Since

$$
\frac{m_{n}\left(D_{t}\right)}{m_{n}(D)}=\frac{m_{n}\left(h\left(D_{t}\right)\right)}{m_{n}(h(D))}
$$

for all $h \in S M\left(\mathbb{R}^{n}\right)$, (7) implies the inequality

$$
\frac{1}{m_{n}\left(h\left(D_{t}\right)\right)} \int_{h\left(D_{t}\right)} u(y) d m_{n}(y) \leq \frac{t}{m_{n}(h(D))} \int_{h(D)} u(y) d m_{n}(y) .
$$

Thus, (5) holds for all $h \in \operatorname{Sim}\left(p_{D}, x_{\Omega}\right)$ with $C=t K$.

REMARK 2.3.1. If $\operatorname{Sim}\left(p_{D}, x_{\Omega}\right)=\emptyset$ for all $x_{\Omega}$, then Proposition 2.3 is vacuously true.

Let $\varphi: S M\left(\mathbb{R}^{n}\right) \rightarrow(0, \infty)$ be a function such that the equality

$$
\varphi(h)=\varphi(i s \circ h)
$$

holds for all $h \in S M\left(\mathbb{R}^{n}\right)$ and for all isometries is $: \mathbb{R}^{n} \rightarrow \mathbb{R}^{n}$. Then, we have $\varphi\left(h_{1}\right)=$ $\varphi\left(h_{2}\right)$ whenever $k\left(h_{1}\right)=k\left(h_{2}\right)$, that is there is a function $f:(0, \infty) \rightarrow(0, \infty)$ such that the equality

$$
\varphi(h)=f(k(h))
$$

is fulfilled for all $h \in S M\left(\mathbb{R}^{n}\right)$ with $k(h)$ equals the similarity constant of $h$. For instance, if $D$ is a bounded non-void subset of $\mathbb{R}^{n}$, we can put $\varphi(h)=\operatorname{diam}(h(D))$. Other examples can be found in the final section of the paper.

Let $D$ be a measurable subset of $\mathbb{R}^{n}$ with a marked point $p_{D} \in$ Int $D$. For every open set $\Omega \subseteq \mathbb{R}^{n}$, define a subset $Q(f, D, \Omega) \subseteq \mathcal{L}_{\text {loc }}^{1}(\Omega)$ by the rule:

$u \in Q(f, D, \Omega)$ if and only if $u \geq 0$ and $u \in \mathcal{L}_{\text {loc }}^{1}(\Omega)$ and there is $K=K(u) \geq 1$ such that the inequality

$$
u\left(x_{\Omega}\right) \leq \frac{K}{(f(k(h)))^{n}} \int_{h(D)} u(y) d m_{n}(y)
$$

holds for every $x_{\Omega} \in \Omega$ and all $h \in \operatorname{Sim}\left(p_{D}, x_{\Omega}\right)$. 
It is clear that $Q N S(\Omega)=Q(f, D, \Omega)$ if $D$ satisfies the conditions of Theorem 2.2 and $f(k(h))=k(h)\left(m_{n}(D)\right)^{\frac{1}{n}}$.

Proposition 2.4. Let $D$ be a bounded, Lebesgue measurable subset of $\mathbb{R}^{n}$ with $a$ marked point $p_{D} \in$ Int $D$ and let $\varphi: S M\left(\mathbb{R}^{n}\right) \rightarrow(0, \infty), f:(0, \infty) \rightarrow(0, \infty)$ be functions such that (8) holds for all $h \in S M\left(\mathbb{R}^{n}\right)$. Then, the inclusion

$$
Q N S(\Omega) \subseteq Q(f, D, \Omega)
$$

is valid for all open sets $\Omega \subseteq \mathbb{R}^{n}$ if and only if there is $c \geq 1$ such that the inequality

$$
f(k) \leq c k
$$

holds for all $k \in(0, \infty)$.

Proof. Suppose that inclusion (10) holds for all open sets $\Omega \subseteq \mathbb{R}^{n}$. Let $\Omega$ be an open half-space of $\mathbb{R}^{n}$. Then, for every $k_{0} \in(0, \infty)$, there is a similarity $h_{0}$ with the similarity constant $k\left(h_{0}\right)=k_{0}$ such that $h_{0}(D) \subseteq \Omega$. The constant function $u_{1}, u_{1}(x) \equiv 1$ for $x \in \Omega$, belongs to $Q N S(\Omega)$. Hence, by (10), $u_{1} \in Q(f, D, \Omega)$ and it follows from (9) that

$$
1=u_{1}\left(h_{0}\left(p_{D}\right)\right) \leq \frac{K}{\left(f\left(k_{0}\right)\right)^{n}} \int_{h_{0}(D)} u_{1}(x) d m_{n}(x)=\frac{K m_{n}\left(h_{0}(D)\right)}{\left(f\left(k_{0}\right)\right)^{n}}=\frac{K\left(k_{0}\right)^{n} m_{n}(D)}{\left(f\left(k_{0}\right)\right)^{n}} .
$$

Consequently (10) implies (11) for all $k \in(0, \infty)$ with

$$
c=\left(K\left(u_{1}\right) m_{n}(D)\right)^{\frac{1}{n}} \vee 1 .
$$

Conversely suppose that (11) holds for all $k \in(0, \infty)$. Then, using Theorem 2.2 , we obtain the following inequalities for every open set $\Omega \subseteq \mathbb{R}^{n}$, every $u \in Q N S(\Omega)$, every $x_{\Omega} \in \Omega$ and every $h \in \operatorname{Sim}\left(p_{D}, x_{\Omega}\right)$ :

$$
\begin{aligned}
u\left(x_{\Omega}\right) & \leq \frac{C(u)}{m_{n}(h(D))} \int_{h(D)} u(x) d m_{n}(x)=\frac{C(u)(f(k(h)))^{n}}{(k(h))^{n} m_{n}(D)(f(k(h)))^{n}} \int_{h(D)} u(x) d m_{n}(x) \\
& \leq \frac{C(u) c^{n}}{m_{n}(D)(f(k(h)))^{n}} \int_{h(D)} u(x) d m_{n}(x) .
\end{aligned}
$$

Hence (9) holds with

$$
K=\frac{C(u) c^{n}}{m_{n}(D)} \vee 1
$$

Thus, (10) is valid for all open sets $\Omega \subseteq \mathbb{R}^{n}$.

Before passing to the equality

$$
Q(f, D, \Omega)=Q N S(\Omega)
$$

we consider one relevant question.

THEOREM 2.5. Let $A$ be a subset of $(0, \infty)$. Then, $A$ is favourable for all open sets $\Omega \subseteq \mathbb{R}^{n}$ if and only if the following statement holds. 
(s) There exists $C=C(A)>1$ such that

$$
\left[\frac{x}{C}, x\right] \cap A \neq \emptyset
$$

for every $x \in(0, \infty)$.

The following lemma will be used in the proof of Theorem 2.5.

Lemma 2.6. Let $A \subseteq(0, \infty)$. Statement $(s)$ of Theorem 2.5 does not hold with this $A$, if and only if there are disjoint open intervals $\left(a_{m}, b_{m}\right), a_{m}<b_{m}, m=1,2, \ldots$, in $(0, \infty) \backslash A$ such that

$$
\lim _{m \rightarrow \infty} \frac{a_{m}}{b_{m}}=0
$$

and either

$$
\lim _{m \rightarrow \infty} a_{m}=\lim _{m \rightarrow \infty} b_{m}=0
$$

or

$$
\lim _{m \rightarrow \infty} a_{m}=\lim _{m \rightarrow \infty} b_{m}=\infty
$$

Proof. If statement (s) holds, then using (12), we obtain that

$$
\frac{a}{b} \geq \frac{1}{C(A)}
$$

for every open interval $(a, b)$ in $(0, \infty) \backslash A$. This inequality contradicts (13).

Conversely, suppose that statement (s) of Theorem 2.5 does not hold and that 0 and $\infty$ are limit points of $A$. Then, for every natural $i \geq 2$, there is $x \in(0, \infty)$ such that

$$
\left(\frac{x}{i}, x\right) \cap A=\emptyset \text {. }
$$

Let $\bar{A}$ be the closure of $A$ in $(0, \infty)$. Write $\left(a_{i}, b_{i}\right)$ for the connected component of $(0, \infty) \backslash$ $\bar{A}$ which contains $\left(\frac{x}{i}, x\right)$. Since both 0 and $\infty$ are the limit points of $A$ we have

$$
0<a_{i}<b_{i}<\infty
$$

Passing to convergent, in $[0, \infty]$, subsequences $\left\{a_{i_{m}}\right\}_{m \in \mathbb{N}}$ and $\left\{b_{i_{m}}\right\}_{m \in \mathbb{N}}$, it is easy to see that limits $\lim _{m \rightarrow \infty} a_{i_{m}}$ and $\lim _{m \rightarrow \infty} b_{i_{m}}$ are 0 or $\infty$ and that the equalities

$$
\lim _{m \rightarrow \infty} a_{i_{m}}=0 \text { and } \lim _{m \rightarrow \infty} b_{i_{m}}=\infty
$$

cannot be true simultaneously. Renaming $a_{m}:=a_{i_{m}}$ and $b_{m}:=b_{i_{m}}$, we obtain the desirable sequence of intervals in $(0, \infty) \backslash A$.

If at least one of the points 0 and $\infty$ is not a limit point of $A$, then there is $\varepsilon>0$ such that

$$
A \subset(0, \varepsilon] \text { or } A \subset[\varepsilon, \infty)
$$


Each of these inclusions implies evidently the existence of desired intervals in $(0, \infty) \backslash \bar{A}$.

Proof of Theorem 2.5. We shall first prove that $A$ is favourable for all open sets $\Omega \subseteq \mathbb{R}^{n}$ if statement (s) holds.

Suppose that (s) is true. Let $\Omega$ be an open set in $\mathbb{R}^{n}$ and let $u \in \mathcal{L}_{\text {loc }}^{1}(\Omega)$ be a nonnegative function, which satisfies condition (ii) of Definition 1.4. It is enough to show that $u \in Q N S(\Omega)$. To prove this, consider an arbitrary $\overline{B^{n}\left(x_{\Omega}, r_{0}\right)} \subseteq \Omega$. By statement (s), there is $r_{1} \in A$ such that

$$
\frac{r_{0}}{C} \leq r_{1} \leq r_{0}
$$

where the constant $C=C(A)>1$. Using this double inequality and condition (ii) of Definition 1.4, we obtain

$$
u\left(x_{\Omega}\right) \leq \frac{K}{v_{n}\left(r_{1}\right)^{n}} \int_{B^{n}\left(x_{\Omega}, r_{1}\right)} u(y) d m_{n}(y) \leq \frac{K C^{n}}{v_{n}\left(r_{0}\right)^{n}} \int_{B^{n}\left(x_{\Omega}, r_{0}\right)} u(y) d m_{n}(y) .
$$

Statement (iv) of Proposition 1.3 implies that $u \in Q N S(\Omega)$.

Conversely, suppose that $A$ is favourable for every open set $\Omega \subseteq \mathbb{R}^{n}$. We must show that (s) holds. If (s) does not hold then, by Lemma 2.6 there is a sequence of disjoint open intervals in $(0, \infty)$ satisfying (13) and (14) or (13) and (15). Suppose that (13) and (14) hold. Then, for every integer $N_{0}>2$, there is a sequence of open intervals $\left(a_{m}, b_{m}\right)$ such that

$$
0<b_{m+1}<a_{m}<2 a_{m}<\frac{1}{N_{0}} b_{m}<b_{m}
$$

and

$$
\left(a_{m}, b_{m}\right) \cap A=\emptyset
$$

for $m=1,2, \ldots$ and

$$
\lim _{m \rightarrow \infty} \frac{b_{m}}{a_{m}}=\infty .
$$

Moreover, passing, if necessary, to a subsequence we may assume that

$$
\sum_{m=1}^{\infty} b_{m}<\infty
$$

For the sake of simplicity, we shall describe our constructions only on the plane but in such a way that a generalisation to the dimensions $n \geq 3$ is a trivial matter.

Define the points $z_{m} \in \mathbb{C}, m=1,2, \ldots$, as

$$
z_{m}:= \begin{cases}0 & \text { if } m=1 \\ 2 \sum_{i=1}^{m-1} b_{i} & \text { if } m \geq 2\end{cases}
$$

and write

$$
R_{1}:=\left\{z \in \mathbb{C}: 0<\operatorname{Re}(z)<2 b_{1},|\operatorname{Im}(z)|<a_{2}\right\}
$$




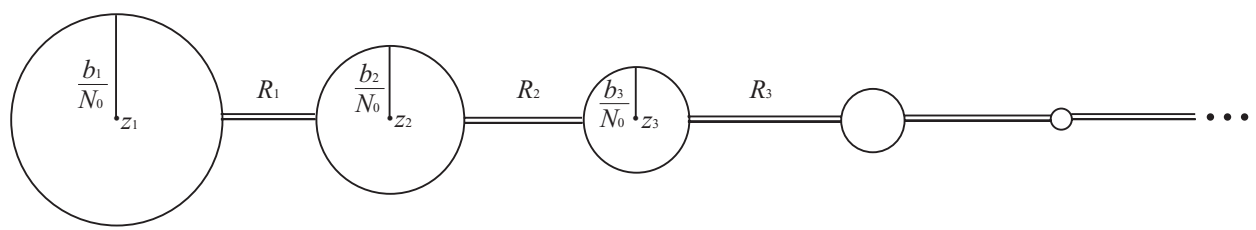

Figure 1. Domain $\Omega$ is an infinite sequence of balls $B^{2}\left(z_{m}, \frac{b_{m}}{N_{0}}\right)$ united by the thin rectangles $R_{m}$.

and

$$
R_{m}:=\left\{z \in \mathbb{C}: 2 \sum_{i=1}^{m-1} b_{i}<\operatorname{Re}(z)<2 \sum_{i=1}^{m} b_{i},|\operatorname{Im}(z)|<a_{m+1}\right\}
$$

for $m \geq 2$. Using (16), we see that $B^{2}\left(z_{m}, b_{m}\right)$ are open, pairwise disjoint balls and that $R_{m}$ are open, pairwise disjoint rectangles. The desired domain $\Omega$ is, by definition, the union

$$
\bigcup_{m=1}^{\infty}\left(B^{2}\left(z_{m}, \frac{b_{m}}{N_{0}}\right) \cup R_{m}\right)
$$

(see Figure 1). Let us define now a function $u$ as the characteristic function of the set

$$
X:=\bigcup_{m=1}^{\infty} \overline{B^{2}\left(z_{m}, a_{m}\right)}
$$

that is

$$
u(z):= \begin{cases}1 & \text { if } z \in X \\ 0 & \text { if } z \in \Omega \backslash X .\end{cases}
$$

It is clear that $u \geq 0$ and that $u \in \mathcal{L}^{1}(\Omega)$. Moreover, since

$$
\frac{1}{m_{2}\left(B^{2}\left(z_{m}, \frac{b_{m}}{2 N_{0}}\right)\right)} \int_{B^{2}\left(z_{m}, \frac{b_{m}}{2 N_{0}}\right)} u(z) d m_{2}(z)=\frac{4 N_{0}^{2}\left(a_{m}\right)^{2}}{\left(b_{m}\right)^{2}},
$$

statement (iv) of Proposition 1.3 and limit relation (18) imply $u \notin Q N S(\Omega)$. It remains to show that there is $K$ such that (3) holds whenever $r \in A$ and $\overline{B^{2}(x, r)} \subseteq \Omega$. If $x \in \Omega \backslash X$, then (3) is trivial and we must consider only $x \in X$. The last membership relation implies that there exists $m=m_{x}$ such that

$$
x \in \overline{B^{2}\left(z_{m}, a_{m}\right)} .
$$

Let us consider all $r \in A$ such that

$$
\overline{B^{2}(x, r)} \subseteq \Omega
$$

From (17) follows that either $r \geq b_{m_{x}}$ or $r \leq a_{m_{x}}$. If $r \geq b_{m_{x}}$, then we have

$$
B^{2}(x, r) \supseteq \overline{B^{2}\left(z_{m_{x}}, \frac{b_{m_{x}}}{N_{0}}\right)} .
$$


Indeed, the triangle inequality and (16) imply

$$
|y-x| \leq\left|x-z_{m_{x}}\right|+\left|z_{m_{x}}-y\right| \leq a_{m_{x}}+\frac{b_{m_{x}}}{N_{0}}<\frac{3}{4} b_{m_{x}}<r
$$

for all $y \in \overline{B^{2}\left(z_{m_{x}}, \frac{b_{m_{x}}}{N_{0}}\right)}$. Inclusion (25) and definition of $\Omega$ show that $B^{2}(x, r) \nsubseteq \Omega$ if $r \geq$ $b_{m_{x}}$. Consequently, if (24) holds, then

$$
r \leq a_{m_{x}} .
$$

Using the last inequality, (23) and (16) we obtain

$$
\overline{B^{2}(x, r)} \subseteq B^{2}\left(z_{m}, \frac{b_{m}}{N_{0}}\right), \quad m=m_{x},
$$

for these $x$ and $r$. Hence, the equality

$$
\frac{1}{m_{2}\left(B^{2}(x, r)\right)} \int_{B^{2}(x, r)} u(y) d m_{2}(y)=\frac{m_{2}\left(B^{2}\left(z_{m}, a_{m}\right) \cap B^{2}(x, r)\right)}{m_{2}\left(B^{2}(x, r)\right)}
$$

holds for such $x$ and $r$. Write

$$
C=\inf \frac{m_{2}\left(B^{2}\left(z_{m}, a_{m}\right) \cap B^{2}(x, r)\right)}{m_{2}\left(B^{2}(x, r)\right)},
$$

where the infimum is taken over the set of all balls $B^{2}(x, r)$ with $x \in B^{2}\left(z_{m}, a_{m}\right)$ and with $r \leq a_{m}$. If $r$ is fixed and $x_{1}, x_{2} \in B^{2}\left(z_{m}, a_{m}\right)$, then the inequality $\left|x_{1}-z_{m}\right| \geq\left|x_{2}-z_{m}\right|$ implies

$$
m_{2}\left(B^{2}\left(z_{m}, a_{m}\right) \cap B^{2}\left(x_{1}, r\right)\right) \leq m_{2}\left(B^{2}\left(z_{m}, a_{m}\right) \cap B^{2}\left(x_{2}, r\right)\right),
$$

(see Figure 2). Thus, we have

$$
C=\inf _{r \leq a_{m}} \frac{m_{2}\left(B^{2}\left(z_{m}, a_{m}\right) \cap B^{2}\left(z_{m}+a_{m}, r\right)\right)}{m_{2}\left(B^{2}\left(z_{m}+a_{m}, r\right)\right)} .
$$

The right-hand side of the last formula is invariant under the similarities. Consequently, using the similarity

$$
\mathbb{C} \ni z \longmapsto \frac{1}{r}\left(z-z_{m}\right) \in \mathbb{C}
$$

we see that

$$
\begin{aligned}
C & =\inf _{r \leq a_{m}} \frac{m_{2}\left(B^{2}\left(0, \frac{a_{m}}{r}\right) \cap B^{2}\left(\frac{a_{m}}{r}, 1\right)\right)}{m_{2}\left(B^{2}\left(\frac{a_{m}}{r}, 1\right)\right)}=\inf _{r \geq 1} \frac{m_{2}\left(B^{2}(0, r) \cap B^{2}(r, 1)\right)}{m_{2}\left(B^{2}(r, 1)\right)} \\
& =\frac{1}{\pi} \inf _{r \geq 1} m_{2}\left(B^{2}(0, r) \cap B^{2}(r, 1)\right)=\frac{1}{\pi} m_{2}\left(B^{2}(-1,1) \cap B^{2}(0,1)\right)=\frac{2}{3}-\frac{\sqrt{3}}{2 \pi} .
\end{aligned}
$$

The last equality, (26) and (28) imply that

$$
\frac{1}{\left(\frac{2}{3}-\frac{\sqrt{3}}{2 \pi}\right) m_{2}\left(B^{2}(x, r)\right)} \int_{B^{2}(x, r)} u_{3}(y) d m_{2}(y) \geq u_{3}(x),
$$




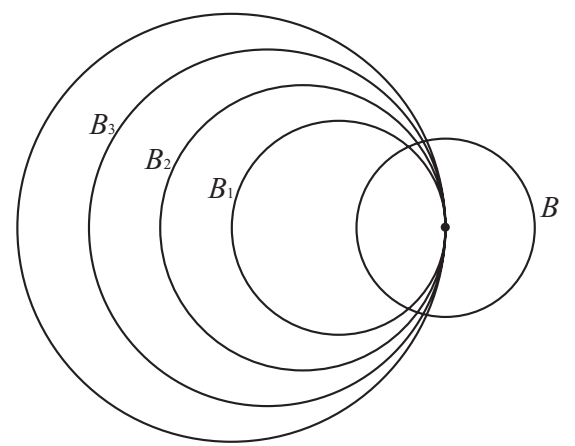

Figure 2. The centre of the fixed small ball $B$ lies on the boundary spheres of the large balls $B_{n}$. The volume of the intersection $B \cap B_{n}$ grows together with the radius of the ball $B_{n}$.

whenever $r \in A$ and $\overline{B^{2}(x, r)} \subseteq \Omega$.

Thus, the theorem is proved in the case, where limit relations (13) and (14) hold. Similar constructions can be realised if (13) and (15) hold and we omit them here.

Statement (s) of Theorem 2.5 has a useful reformulation. For $A \subseteq(0, \infty)$ define

$$
\ln (A):=\{\ln x: x \in A\}
$$

with $\ln (\emptyset):=\emptyset$. Then, $\ln (A)$ is a subset of $\mathbb{R}$. Recall that a set $X \subseteq \mathbb{R}$ is an $\varepsilon$-net in $\mathbb{R}$, $\varepsilon>0$, if

$$
\mathbb{R}=\bigcup_{x \in X} B^{1}(x, \varepsilon)
$$

Proposition 2.7. Let $A$ be a subset of $(0, \infty)$. Then, statement $(s)$ in Theorem 2.5 is valid with this $A$ if and only if there is $\varepsilon>0$ such that $\ln (A)$ is an $\varepsilon$-net in $\mathbb{R}$.

Proof. If (s) holds, then $\ln (A)$ is an $\varepsilon$-net with $\varepsilon=\ln C$, where $C$ is the constant in (12). If (s) does not hold, then Lemma 2.6 implies that $\ln A$ is not an $\varepsilon$-net for any $\varepsilon>0$.

Using this proposition and analysing the first part of the proof of Theorem 2.5 , we obtain the following.

Proposition 2.8. Let $A$ be a subset of $(0, \infty)$. The following three statements are equivalent.

(i) $A$ is favourable for all domains of $\mathbb{R}^{n}$.

(ii) $A$ is favourable for all open sets of $\mathbb{R}^{n}$.

(iii) There is $\varepsilon>0$ such that $\ln (A)$ is an $\varepsilon$-net in $\mathbb{R}$.

The condition for the set $A \subseteq(0, \infty)$, to be favourable for all bounded domains $\Omega$ can be presented in terms of porosity of $A$, so recall a definition. 
Definition 2.9. Let $A \subseteq(0, \infty)$. The right-hand porosity of $A$ at zero is the quantity

$$
p_{0}(A):=\limsup _{h \rightarrow 0+} \frac{l(h, A)}{h},
$$

where $l(h, A)$ is the length of the longest interval in $[0, h] \backslash A, h>0$.

REMARK 2.9.1. It is easy to see that $0 \leq p_{0}(A) \leq 1$ for each $A \subseteq \mathbb{R}$. A variety of computations directly related to the notion of porosity can be found in [19, pp. 183-212].

Definition 2.10. Let $A \subseteq(0, \infty)$. The right porosity index of $A$ at $0, i_{0}(A)$, is defined to be the supremum of all real numbers $r$ for which there is a sequence of open intervals $\left\{\left(a_{n}, b_{n}\right)\right\}_{n \in \mathbb{N}}, a_{n}<b_{n}$, such that $\lim _{n \rightarrow \infty} a_{n}=\lim _{n \rightarrow \infty} b_{n}=0$ and $\left(a_{n}, b_{n}\right) \subset$ $(0, \infty) \backslash A$ and

$$
r<\frac{b_{n}-a_{n}}{a_{n}}
$$

for each $n \in \mathbb{N}$.

If no such numbers $r$ exist, then following the usual conversion we define $i_{0}(A):=0$.

The following lemma is a particular case of Lemma $A_{2.13}$ from [19, p. 185].

LEMMA 2.11. The equality

$$
i_{0}(A)=\frac{p_{0}(A)}{1-p_{0}(A)}
$$

holds for each $A \subseteq(0, \infty)$.

THEOREM 2.12. Let $A$ be a subset of $(0, \infty)$. Then, $A$ is favourable for all bounded domains $\Omega \subseteq \mathbb{R}^{n}$ if and only if $p_{0}(A)<1$.

Proof. It follows from Lemma 2.11 that $p_{0}(A)=1$ if and only if $i_{0}(A)=\infty$. Using the definition of porosity index $i_{0}(A)$, we can prove that the equality $i_{0}(A)=\infty$ implies the existence of disjoint intervals $\left(a_{n}, b_{n}\right) \subset(0, \infty) \backslash A, n=1,2, \ldots$, such that equations (13) and (14) hold. It was shown in the proof of Theorem 2.5 that if (13) and (14) hold, then there are a domain $\Omega \subseteq \mathbb{R}^{n}$ and a non-negative $u \in \mathcal{L}_{\text {loc }}^{1}(\Omega) \backslash Q N S(\Omega)$ such that (3) holds whenever $r \in A$ and $\overline{B^{n}(x, r)} \subseteq \Omega$. It remains to observe that inequality (19) implies $\operatorname{diam}(\Omega)<\infty$. Thus, if $A$ is favourable for all bounded domains $\Omega \subseteq \mathbb{R}^{n}$, then $p_{0}(A)<1$.

Now note that if $p_{0}(A)<1$, then the set $(-\infty, R) \cap \ln (A)$ is an $\varepsilon$-net, $\varepsilon=\varepsilon(R)$, in $(-\infty, R)$ for each $R \in \mathbb{R}$. Hence, reasoning as in the first part of the proof of Theorem 2.5, we can prove the implication

$$
\left(p_{0}(A)<1\right) \Rightarrow\left(A \text { is favourable for every bounded domain } \Omega \subseteq \mathbb{R}^{n}\right) .
$$

REMARK 2.12.1. As in Proposition 2.8, it is easy to prove that $A$ is favourable for all bounded domains of $\mathbb{R}^{n}$ if and only if $A$ is favourable for all bounded open subsets of $\mathbb{R}^{n}$. Theorem 2.12 remains valid even for unbounded domains and open sets $\Omega \subseteq \mathbb{R}^{n}$ if

$$
\sup _{x \in \Omega}\left(\delta_{\Omega}(x)\right)<\infty
$$


REMARK 2.12.2. In complete analogy with Definition 2.10, we may define the quantity $i_{\infty}(A)$, the left porosity index of $A$ at $+\infty$, after which Proposition 2.8 can be reformulated as:

Let $A \subset(0, \infty)$. The following statements are equivalent:

(i) $A$ is favourable for all domains $\Omega \subseteq \mathbb{R}^{n}$;

(ii) The indexes $i_{0}(A)$ and $i_{\infty}(A)$ are less than infinity,

$$
i_{0}(A) \vee i_{\infty}(A)<\infty
$$

(iii) There is $\varepsilon>0$ such that $\ln (A)$ is an $\varepsilon$-net in $\mathbb{R}^{1}$.

Theorem 2.5, Proposition 2.7 and Theorem 2.12 imply the following.

COROllary 2.13. Let $A$ be a subset of $(0, \infty)$ and let $\alpha, \beta$ be positive constants. Then, the set $A$ is favourable for all domains $\Omega \subseteq \mathbb{R}^{n}$ (for all bounded domains $\Omega \subseteq \mathbb{R}^{n}$ ) if and only if the set

$$
\alpha A^{\beta}:=\left\{\alpha x^{\beta}: x \in A\right\}
$$

has the same property.

Proof. One just directly observe that if condition (s) holds for the set $A$ with a constant $C$, then condition (s) holds for the set $\alpha A^{\beta}$ with the constant $C^{\prime} \geq C^{\beta}$.

Now, we are ready to characterise the function $f:(0, \infty) \rightarrow(0, \infty)$ for which the equality

$$
Q(f, D, \Omega)=Q N S(\Omega)
$$

is fulfilled for all open sets $\Omega \subseteq \mathbb{R}^{n}$.

THEOREM 2.14. Let $D$ be a bounded, Lebesgue measurable subset of $\mathbb{R}^{n}$ with a marked point $p_{D} \in$ Int $D$ and let $\varphi: S M\left(\mathbb{R}^{n}\right) \rightarrow(0, \infty), f:(0, \infty) \rightarrow(0, \infty)$ be functions such that (8) holds for all $h \in S M\left(\mathbb{R}^{n}\right)$. Then, equality (30) holds for all open sets $\Omega \subseteq \mathbb{R}^{n}$ if and only if there are $A \subseteq(0, \infty)$ and $c>1$ such that:

(i) the inequality $f(k) \leq c k$ holds for all $k \in(0, \infty)$,

(ii) $\ln (A)$ is an $\varepsilon$-net in $\mathbb{R}$ for some $\varepsilon>0$,

(iii) the inequality

$$
\frac{1}{c} k \leq f(k)
$$

holds for all $k \in A$.

Proof. Let $\Omega$ be an open set in $\mathbb{R}^{n}, A \subseteq(0, \infty)$ and $c>1$. Assume that $\ln (A)$ is an $\varepsilon$-net in $\mathbb{R}$ for some $\varepsilon>0$ and that (31) holds with this $c$ for all $k \in A$. Then, using (9) and (31), we obtain

$$
u\left(x_{\Omega}\right) \leq \frac{c^{n} K(u)}{(k(h))^{n}} \int_{h(D)} u(y) d m_{n}(y)
$$


for every $u \in Q(f, D, \Omega)$ and every $x_{\Omega}$ whenever $h \in \operatorname{Sim}\left(p_{D}, x_{\Omega}\right)$ and $k(h) \in A$. As in the proof of Theorem 2.2 write

$$
R_{D}=\sup _{y \in D}\left|p_{D}-y\right|
$$

Let $B^{n}\left(x_{\Omega}, r_{0}\right)$ be a ball such that $r_{0}=R_{D} k_{0}$, with $k_{0} \in A$ and $\overline{B^{n}\left(x_{\Omega}, r_{0}\right)} \subset \Omega$. Then, each similarity $h$ such that $k(h)=k_{0}$ and $h\left(p_{D}\right)=x_{\Omega}$ belongs to $\operatorname{Sim}\left(p_{D}, x_{\Omega}\right)$ and satisfies $h(D) \subseteq B^{n}\left(x_{\Omega}, r_{0}\right)$. Consequently, (32) implies

$$
u\left(x_{\Omega}\right) \leq \frac{c^{n} K(u)\left(R_{D}\right)^{n} v_{n}}{\left(k_{0} R_{D}\right)^{n} v_{n}} \int_{B^{n}\left(x_{\Omega}, r_{0}\right)} u(y) d m_{n}(y)=\frac{c^{n} K(u) v_{n}\left(R_{D}\right)^{n}}{m_{n}\left(B^{n}\left(x_{\Omega}, r_{0}\right)\right)} \int_{B^{n}\left(x_{\Omega}, r_{0}\right)} u(y) d m_{n}(y)
$$

for every $u \in Q(f, D, \Omega)$ and every $B^{n}\left(x_{\Omega}, r_{0}\right) \subseteq \Omega$ whenever $r_{0} \in R_{D} A$. Corollary 2.13 implies that the set $R_{D} A$ is favourable for $\Omega$. Hence, $Q(f, D, \Omega) \subseteq Q N S(\Omega)$. Taking into account Proposition 2.4, we see that conditions (i)-(iii) of the present theorem imply equality (30) for all open sets $\Omega \subseteq \mathbb{R}^{n}$.

Conversely, suppose that (30) holds for all open sets $\Omega \subseteq \mathbb{R}^{n}$ but for every $t>0$ the set $\ln A_{t}$, where

$$
A_{t}:=\left\{k \in(0, \infty): f(k) \geq \frac{1}{t} k\right\}
$$

is not an $\varepsilon$-net for any $\varepsilon>0$. It is clear that $A_{t_{1}} \subseteq A_{t_{2}}$ if $t_{1} \geq t_{2}$. Applying Proposition 2.7 and Lemma 2.6 to the sets $A_{2}, A_{3}, \ldots$, we obtain a sequence $\left\{a_{m}\right\}_{m=2}^{\infty}$ of positive numbers $a_{m}$ such that $A_{m} \cap\left(a_{m}, m a_{m}\right)=\emptyset$ for each $m \geq 2$, that is

$$
f(k)<\frac{1}{m} k
$$

if $a_{m}<k<m a_{m}$, and that

$$
\left(a_{m_{1}}, m_{1} a_{m_{1}}\right) \cap\left(a_{m_{2}}, m_{2} a_{m_{2}}\right)=\emptyset,
$$

whenever $m_{1} \neq m_{2}$. Passing, if necessary, to a subsequence we may assume that $\left\{a_{m}\right\}_{m=2}^{\infty}$ and $\left\{m a_{m}\right\}_{m=2}^{\infty}$ are monotone and convergent in $[0, \infty]$ sequences. This assumption and (35) imply either the equalities

$$
\lim _{m \rightarrow \infty} a_{m}=\lim _{m \rightarrow \infty} m a_{m}=0
$$

or the equalities

$$
\lim _{m \rightarrow \infty} a_{m}=\lim _{m \rightarrow \infty} m a_{m}=\infty
$$

As in the proof of Theorem 2.5, we consider only the case when (36) holds and the dimension $n=2$. We shall construct a domain $\Omega \subseteq \mathbb{R}^{2}$ and a non-negative $u \in \mathcal{L}_{\text {loc }}^{1}(\Omega)$ such that

$$
u \in Q(f, D, \Omega) \backslash Q N S(\Omega) .
$$

To this end note that (30) implies (10), so using Proposition 2.4 we can find $c \geq 1$ such that

$$
f(k) \leq c k
$$


for every $k \in(0, \infty)$. Let us define a function $f_{1}:(0, \infty) \rightarrow(0, \infty)$ by the rule

$$
f_{1}(k):=\left\{\begin{array}{ll}
\frac{k}{m} & \text { if } a_{m}<k<m a_{m}, m=2,3, \ldots \\
c k & \text { if } k \in(0, \infty) \backslash \bigcup_{m=2}^{\infty}\left(a_{m}, m a_{m}\right)
\end{array},\right.
$$

where $c \geq 1$ is the constant from inequality (37). Inequalities (34) and (37) imply $f(k) \leq$ $f_{1}(k)$ for all $k \in(0, \infty)$. Hence, from the definition of the set $Q(f, D, \Omega)$ follows the inclusion

$$
Q(f, D, \Omega) \supseteq Q\left(f_{1}, D, \Omega\right) .
$$

Thus, it is sufficient to find a domain $\Omega \subseteq \mathbb{R}^{2}$ and a non-negative $u \in \mathcal{L}_{\text {loc }}^{1}(\Omega)$ such that

$$
u \in Q\left(f_{1}, D, \Omega\right) \backslash Q N S(\Omega) .
$$

Let us define

$$
\Omega:=\bigcup_{m=N_{0}+1}^{\infty}\left(B^{2}\left(z_{m}, \frac{m a_{m}}{N_{0}}\right) \cup R_{m}\right), \quad u(x):=\left\{\begin{array}{lll}
1 & \text { if } & x \in X \\
0 & \text { if } & x \in \Omega \backslash X
\end{array},\right.
$$

where

$$
\begin{gathered}
X:=\bigcup_{m=N_{0}+1}^{\infty} \overline{B^{2}\left(z_{m}, a_{m}\right)}, \quad z_{m}:=2 \sum_{i=1}^{m-1} i a_{i}, \\
R_{m}:=\left\{z \in \mathbb{C}: 2 \sum_{n=1}^{m-1} n a_{n}<\operatorname{Re}(z)<2 \sum_{n=1}^{m} n a_{n}, \quad|\operatorname{Im}(z)|<a_{m+1}\right\} .
\end{gathered}
$$

The parameter $N_{0}$ is free here and we will specify this parameter later. It is relevant to remark that the domain $\Omega$ is obtained from the domain depicted on Figure 1, by deleting of the balls $B^{2}\left(z_{1}, \frac{b_{1}}{N_{0}}\right), B^{2}\left(z_{2}, \frac{b_{2}}{N_{0}}\right), \ldots, B^{2}\left(z_{N_{0}}, \frac{b_{N_{0}}}{N_{0}}\right)$ and the rectangles $R_{1}, \ldots, R_{N_{0}}$ and putting $b_{m}:=m a_{m}$ in the rest of balls and rectangles. As in the proof of Theorem 2.5, we have $u \notin Q N S(\Omega)$. It still remains to prove that $u \in Q\left(f_{1}, D, \Omega\right)$. The last relation holds if and only if there exists $K(u) \geq 1$ such that

$$
\left(f_{1}(k(h))\right)^{2} \leq K(u) \int_{h(D)} u(y) d m_{2}(y)
$$

for all $h \in \operatorname{Sim}\left(p_{D}, x_{\Omega}\right)$ with $x_{\Omega} \in X$.

Let $x_{\Omega} \in X$. It follows from the definitions of $\Omega$ and $X$ that there is $m \geq N_{0}+1$ for which

$$
x_{\Omega} \in \overline{B^{2}\left(z_{m}, a_{m}\right)}
$$

We claim that the inequality

$$
k(h) r_{D} \leq \frac{2 m a_{m}}{N_{0}},
$$

holds for every $h \in \operatorname{Sim}\left(p_{D}, x_{\Omega}\right)$ with $r_{D}=\delta_{\operatorname{Int}(D)}\left(p_{D}\right)$. 
Let us prove it. Since $h \in \operatorname{Sim}\left(p_{D}, x_{\Omega}\right)$, we have

$$
h\left(B^{2}\left(p_{D}, r_{D}\right)\right) \subseteq \Omega .
$$

The last inclusion implies

$$
\partial \Omega \cap h\left(B^{2}\left(p_{D}, r_{D}\right)\right)=\emptyset
$$

because $\Omega \cap \partial \Omega=\emptyset$ for the open sets. The intersection

$$
\partial B^{2}\left(z_{m}, \frac{m a_{m}}{N_{0}}\right) \cap \partial \Omega=\left\{z \in \mathbb{C}:\left|z-z_{m}\right|=\frac{m a_{m}}{N_{0}}\right\} \cap \partial \Omega
$$

is not empty (see Figure 1). Consequently, there is $\xi \in \partial B^{2}\left(z_{m}, \frac{m a_{m}}{N_{0}}\right) \backslash h\left(B^{2}\left(p_{D}, r_{D}\right)\right)$. Hence,

$$
\left|x_{\Omega}-\xi\right| \geq k(h) r_{D}
$$

Using the triangle inequality, we obtain

$$
\left|x_{\Omega}-\xi\right| \leq\left|x_{\Omega}-z_{m}\right|+\left|z_{m}-\xi\right|=\left|x_{\Omega}-z_{m}\right|+\frac{m a_{m}}{N_{0}} .
$$

Consequently,

$$
k(h) r_{D} \leq\left|x_{\Omega}-z_{m}\right|+\frac{m a_{m}}{N_{0}} .
$$

Since $x_{\Omega} \in \overline{B^{2}\left(z_{m}, a_{m}\right)}$, we have $\left|x_{\Omega}-z_{m}\right| \leq a_{m}$. It follows directly from the definition of $\Omega$ that $m \geq N_{0}$. Hence,

$$
k(h) r_{D} \leq a_{m}+\frac{m a_{m}}{N_{0}} \leq \frac{2 m a_{m}}{N_{0}}
$$

Inequality (40) follows.

Since $h(D) \supseteq h\left(B^{2}\left(p_{D}, r_{D}\right)\right)$, the inequality

$$
\left(f_{1}(k(h))\right)^{2} \leq K(u) \int_{B^{2}\left(x_{\Omega}, k(h) r_{D}\right)} u(y) d m_{2}(y)
$$

implies (39), so it is sufficient to prove (41). The following two cases are possible: $k(h) \in$ $\left(0, a_{m}\right]$ and $k(h) \in\left(a_{m}, \infty\right)$. Before analysing these cases note that $f_{1}(k) \leq c k$ for every $k \in(0, \infty)$ because $\frac{1}{m} \leq \frac{1}{2}$ and $c \geq 1$ in definition (38). Hence, in the first case, we can replace (41) by

$$
c^{2} \leq \frac{K(u)}{(k(h))^{2}} \int_{B^{2}\left(x_{\Omega}, k(h) r_{D}\right)} u(y) d m_{2}(y) .
$$

It is clear that

$$
\frac{K(u)}{(k(h))^{2}} \int_{B^{2}\left(x_{\Omega}, k(h) r_{D}\right)} u(y) d m_{2}(y) \geq \frac{K(u) \pi\left(r_{D} \wedge 1\right)^{2}}{\pi\left(k(h)\left(r_{D} \wedge 1\right)\right)^{2}} \int_{B^{2}\left(x_{\Omega}, k(h)\left(r_{D} \wedge 1\right)\right)} u(y) d m_{2}(y) .
$$


Since $k(h) \in\left(0, a_{m}\right]$, we see that

$$
k(h)\left(r_{D} \wedge 1\right) \leq a_{m}
$$

Hence, as it was shown in the proof of Theorem 2.5, in the case under consideration we have

$$
\frac{1}{\pi\left(k(h)\left(r_{D} \wedge 1\right)\right)^{2}} \int_{B^{2}\left(x_{\Omega}, k(h)\left(r_{D}\right) \wedge 1\right)} u(y) d m_{2}(y) \geq \frac{2}{3}-\frac{\sqrt{3}}{2 \pi} .
$$

The last estimation and (43) show that (42) holds if

$$
c^{2}=K(u) \pi\left(r_{D} \wedge 1\right)^{2}\left(\frac{2}{3}-\frac{\sqrt{3}}{2 \pi}\right) .
$$

Consider now the case $k(h) \in\left(a_{m}, \infty\right)$. Inequality (40) shows that

$$
k(h) \leq \frac{2 m a_{m}}{N_{0} r_{D}} .
$$

Let us specify $N_{0}$ as the smallest positive integer $N$ satisfying the inequality $\frac{2}{N r_{D}}>1$. Then, we obtain the double inequality

$$
a_{m}<k(h)<m a_{m} .
$$

This inequality and (38) show that

$$
f_{1}(k(h))=\frac{k}{m} \leq a_{m}
$$

Consequently, we can prove (41) as in the case $k(h) \in\left(0, a_{m}\right]$.

Let us consider now some examples of functions $\varphi$ and $f$ for which equality (8) holds.

EXAMPLE 2.15. Let $\psi$ be a positive bounded periodic function on $\mathbb{R}$. Write

$$
\mu(x):=\frac{1}{2}\left(x+\frac{1}{x}\right)
$$

for $x>0$ and define

$$
f(k):=k \psi(\mu(k))
$$

Using some routine estimations, we see that conditions (i)-(iii) from Theorem 2.14 are satisfied by the function $f$ if we take

$$
A=\mu^{-1}\left\{x \in(0, \infty): \psi(x) \geq \frac{1}{2} M\right\}, \quad c=M \vee \frac{2}{M},
$$

where

$$
M=\sup _{y \in \mathbb{R}} \psi(y) .
$$


An important special case of the preceding example is the constant function $\psi$. Then, $f$ is linear on $(0, \infty)$ and conditions (i)-(iii) from Theorem 2.14 are evidently hold. In this simplest case, the function $\varphi: S M\left(\mathbb{R}^{n}\right) \rightarrow(0, \infty)$ connected with $f$ can be obtained in distinct ways depending on the geometrical properties of the set $D$.

In all following examples, $D$ is a bounded Lebesgue measurable subset of $\mathbb{R}^{n}$ with Int $D \neq \emptyset$ and $h \in S M\left(\mathbb{R}^{n}\right)$.

EXAMPLE 2.16. Let $d$-dimensional Hausdorff measure $\mathcal{H}^{d}, n-1 \leq d \leq n$, of the boundary $\partial D$ be finite and non-zero, $0<\mathcal{H}^{d}(\partial D)<\infty$. Write

$$
\varphi(h)=\left(\mathcal{H}^{d}(\partial(h(D)))\right)^{\frac{1}{d}} .
$$

EXAmple 2.17. Let $D$ be a set with the finite Caccoppoli-de Gorgi perimeter $P$, see for instance [3, Chapter 3, Section 3]. Write

$$
\varphi(h)=(P(h(D)))^{\frac{1}{n-1}} .
$$

EXAMPLE 2.18. Let $D \subseteq \mathbb{R}^{2}$ be a simply connected domain with the rectifiable boundary $\partial D, 0<\mathcal{H}^{1}(\partial D)<\infty$. Suppose that the domain $D$ is not a disk. Write

$$
\varphi(h)=\left(\left(\mathcal{H}^{1}(h(\partial D))\right)^{2}-4 \pi m_{2}(h(D))\right)^{\frac{1}{2}} .
$$

In this case, the inequality $\varphi(h)>0$ follows from the Classical Isoperimetric Inequality, see for instance [3, Chapter 1, Section 1].

This list of examples can be simply extended by involving the analytic capacity, the transfinite diameter, the Menger curvature etc. for the definition of the function $\varphi$. The homogeneity under dilatations $x \longmapsto \alpha x, x \in \mathbb{R}^{n}, \alpha>0$, the invariance under isometries, finiteness and positiveness are sufficient for this purpose.

ACKNOWLEDGEMENT. Oleksiy Dovgoshey was partially supported by the Academy of Finland.

\section{REFERENCES} 2001).

1. D. H. Armitage and S. J. Gardiner, Classical potential theory (Springer-Verlag, London,

2. M. Brelot, Éléments de la théorie classique du potential, second ed. (Centre de Documentation Universitaire, Paris, 1961) .

3. Yu. Burago and V. Zalgaller, Geometric inequalities (Springer-Verlag, New York, 1988). 1968).

4. J. Dieudonne, Algèbre linéaire et géometrie Élémentaire. Troisiéme ed. (Hermann, Paris,

5. O. Djordjević and M. Pavlović, $\mathcal{L}^{p}$-integrability of the maximal function of a polyharmonic function, J. Math. Anal. Appl. 336 (2007), 411-417.

6. O. Dovgoshey and J. Riihentaus, Bi-Lipschitz mappings and quasinearly subharmonic functions, Internat. J. Math. and Math. Sci. (2010), ID382179, 8 p.

7. F. W. Gehring and O. Martio, Quasiextremal distance domains and extension of quasiconformal mappings, J. D'Analyse Mathématique 45 (1985), 181-206.

8. M. Hervé, Analytic and plurisubharmonic functions in finite and infinite dimensional spaces. Lecture Notes in Mathematics, vol. 198 (Springer, Berlin, Heidelberg, 1971).

9. V. Kojić, Quasi-nearly subharmonic functions and conformal mappings. Filomat. 21(2) (2007), 243-249. 
10. M. Pavlović, On subharmonic behavior and oscillation of functions on balls in $\mathbb{R}^{n}$, Publ. Inst. Math. (Beograd) 55(69) (1994), 18-22.

11. M. Pavlović and J. Riihentaus, Classes of quasi-nearly subharmonic functions. Potential Anal. 29 (2008), 89-104.

12. J. Riihentaus, On a theorem of Avanissian-Arsove. Expo. Math. 7 (1989), 69-72.

13. J. Riihentaus, Subharmonic functions: Non-tangential and tangential boundary behavior, in Function spaces, differential operators and nonlinear analysis (FSDONA'99), Proceedings of the syöte conference 1999, (Mustonen V. and Rákosnik J. Editors), Math. Inst. (Czech Acad. Science, Praha, 2000), 229-238.

14. J. Riihentaus, A generalized mean value inequality for subharmonic functions, Expo. Math. 19 (2001), 187-190.

15. J. Riihentaus, A weighted boundary limit result for subharmonic functions, Adv. Algebra and Analysis, 1 (2006), 27-38.

16. J. Riihentaus, Separately quasi-nearly subharmonic functions, in Complex analysis and potential theory, Proceedings of the conference satellite to ICM 2006 (Azeroğlu T. A. and Tamrazov P. M. Editors), Gebze Institute of Technology, Gebze, Turkey, September 8-14, 2006 (World Scientific, Singapore, 2007), 156-165.

17. J. Riihentaus, Subharmonic functions, generalizations and separately subharmonic functions. The XIVth Conference on Analytic Functions, July 22-28, 2007, Chełm, Poland, in Scientific Bulletin of Chetm, Section of Mathematics and Computer Science, 2 (2007), 49-76. (ISBN 978-83-61149-24-8) (arXiv:math/0610259v5 [math.AP] 8 October 2008).

18. J. Riihentaus, On an inequality related to the radial growth of subharmonic functions, Cubo, 11(4) (2009), 127-136.

19. B. S. Thomson, Real functions. Lecture Notes in Mathematics, vol. 1170 (Springer-Verlag, Berlin, Heidelberg, 1985). 